\title{
Anne BAUD et Christian SAPIN, Cluny : les origines $d u$ monastère et de ses églises
}

\section{Didier Méhu}

\section{(2) OpenEdition}

\section{Journals}

Édition électronique

URL : https://journals.openedition.org/ccm/8665

DOI : $10.4000 / \mathrm{ccm} .8665$

ISSN : 2119-1026

\section{Éditeur}

Centre d'études supérieures de civilisation médiévale/Université de Poitiers

\section{Édition imprimée}

Date de publication : 1 décembre 2021

Pagination : $369-373$

ISBN : $978-2-490783-11-3$

ISSN : 0007-9731

Référence électronique

Didier Méhu, "Anne Baud et Christian sapIN, Cluny : les origines du monastère et de ses églises 》, Cahiers de civilisation médiévale [En ligne], 256 | 2021, mis en ligne le 01 décembre 2021, consulté le 25 mai 2022. URL : http://journals.openedition.org/ccm/8665; DOI : https://doi.org/10.4000/ccm.8665

\section{c) $(7)(9)$}

La revue Cahiers de civilisation médiévale est mise à disposition selon les termes de la Licence Creative Commons Attribution - Pas d'Utilisation Commerciale - Pas de Modification 4.0 International. 
Anne BAud et Christian SAPIN, Cluny : les origines du monastère et de ses églises, Walter BerRY, Anne Flammin et Fabrice HenRIon (collab.), Paris, Éditions du CTHS (Collection archéologie et histoire de l'art, 35), 2019.

Le livre codirigé par Anne Baud et Christian Sapin rassemble les résultats des fouilles archéologiques effectuées dans l'abbaye de Cluny entre 2006 et 2013. L'objectif principal était une meilleure compréhension du sanctuaire de l'église dite Cluny II, la seconde église abbatiale identifiée comme telle et datée de la fin du $\mathrm{X}^{\mathrm{e}} \mathrm{s}$. par l'archéologue Kenneth John Conant, lors des fouilles qu'il avait dirigées entre 1936 et 1938 puis en 1949 et 1950. Il s'agissait également de mieux connaître les origines du monastère, voire l'occupation du site avant l'arrivée des moines en 910, dans la mesure où K. J. Conant avait localisé sous l'abside principale de Cluny II une chapelle, qu'il baptisait Cluny A et qu'il interprétait comme le lieu de culte de la villa donnée par le duc d'Aquitaine pour y établir un monastère. Dès 1988, C. Sapin avait émis des doutes sur les interprétations de cette zone par l'archéologue américain, en supposant notamment que Cluny A aurait été la crypte de Cluny II plutôt que l'église de la villa carolingienne. Les fouilles dirigées par A. Baud en 1993 et 1994 sous le transept de l'église abbatiale de Cluny III, situé un peu plus au nord, avaient mis au jour quelques vestiges datant des premières années de l'installation monastique. L'ensemble encourageait à rouvrir entièrement le dossier des origines architecturales de Cluny.

La configuration actuelle du site n'était pourtant pas la plus propice pour mener une telle recherche. Une partie notable du chevet de Cluny II se situe en effet sous les galeries du cloître du XVIII ${ }^{\mathrm{e}} \mathrm{s}$. et sous des bureaux de l'Ecole nationale des arts et métiers où les fouilles ne pouvaient pas être entreprises. Par conséquent, elles furent conduites principalement dans la « galerie rouge », un espace situé entre la galerie orientale du cloître et les bureaux, correspondant à la salle capitulaire de la période gothique, ainsi que dans les jardins situés à l'est des bureaux, où les plans anciens et les fouilles de K. J. Conant avaient notamment localisé une église dédiée à la Vierge (chapelle Sainte-Marie) qui communiquait, sur sa façade ouest, avec la salle capitulaire et dont la construction était présumée dater des premiers temps de l'installation monastique. Une vingtaine de sondages ponctuels furent également effectués dans des zones situées au nord-ouest et au sud-est des bâtiments conventuels du XVIII $\mathrm{s}$. (un plan détaillé des zones fouillées figure p. 11).

Les résultats sont présentés d'une manière chronologico-thématique en 7 chapitres, écrits tantôt par les deux auteurs principaux, tantôt par un de leurs collaborateurs ou avec son aide. Le premier (A. Baud et C. Sapin) présente les sources textuelles et iconographiques qui évoquent l'organisation architecturale du monastère, depuis ses origines jusqu'au milieu du XI ${ }^{\mathrm{e}} \mathrm{s}$. Le lecteur qui n'est pas au fait de la documentation clunisienne sera un peu perdu, car les textes sont présentés très brièvement et, pour plus de détails, il devra encore se reporter au panorama que K. J. Conant en avait dressé dans son ouvrage de synthèse paru en 1968 (Kenneth J. Conant, Cluny : les églises et la maison du chef d'ordre, Cambridge, Mediaeval Academy of America [Publication, 77], 1968) ou aux compléments publiés ultérieurement par Neil Stratford (Neil STRATFORD, « Les bâtiments de l'abbaye de Cluny à l'époque médiévale : état des questions, J. HenRIET [trad.], Bulletin monumental, 150, 1992, p. 383-411, DOI : 10.3406/bulmo.1992.4491). Le second chapitre, dû à Walter Berry, malheureusement décédé peu avant la parution du livre, présente une analyse détaillée des méthodes de fouilles, de relevé et d'enregistrement de K. J. Conant. Alors que la fiabilité de ses interprétations archéologiques et la qualité de son travail sur le terrain ont été vivement critiquées depuis les années 1980, W. Berry montre la rigueur et la précision de ses relevés, ainsi que leur cohérence avec les méthodes de l'archéologie américaine des années 1920-1930, qui était alors à la pointe de la « révolution stratigraphique». $\mathrm{Si}$, dans ses publications, K. J. Conant a très peu évoqué la stratigraphie pour se concentrer sur les 
reconstitutions des édifices en élévation, ses archives permettent de restituer son « registre archéologique». C'est à une telle restitution que W. Berry invite les futurs archéologues de Cluny, afin d'établir les relations entre, d'une part, les vestiges structurels et la stratigraphie observés par K. J. Conant et, d'autre part, le phasage établi par les fouilles récentes. Les chap. 3 et 4 (A. Baud et C. Sapin) rassemblent les résultats relatifs à la villa carolingienne et aux premiers temps du monastère - nous y reviendrons plus loin. Le chap. 5 (C. Sapin et Fabrice Henrion) présente les fouilles dans le secteur de Cluny II. Outre le chevet de l'abbatiale, dont le plan est désormais connu avec une certaine assurance, on retiendra la découverte du soubassement de l'autel de cette église, qui conservait des reliques des saints Pierre et Paul, et dont les différents trous et encoches permettent de restituer la configuration de la fin du $x^{\mathrm{e}}$ et du $\mathrm{XI}^{\mathrm{e}} \mathrm{s}$. L'autel était établi sur quatre piles angulaires et une armoire-reliquaire centrale selon un modèle développé à l'époque paléochrétienne, notamment à Rome. Des panneaux métalliques disposés sur les côtés ornaient le soubassement et fermaient l'accès aux reliques; l'autel était surmonté d'un ciborium. Les chap. 6 (A. Baud et Anne Flammin) et 7 (A. Baud et C. Sapin) rassemblent des découvertes moins novatrices mais précieuses pour établir avec précision l'évolution du complexe monastique autour de la chapelle Sainte-Marie et de la salle capitulaire. On sait désormais que la chapelle Sainte-Marie a été construite à la place de l'ancienne chapelle de la villa (à une date inconnue située au milieu du $\mathrm{x}^{\mathrm{e}} \mathrm{s}$.) et qu'elle a elle-même subi une reconstruction complète située à la fin du XI $\mathrm{e}$ s. pour être clairement articulée à la salle capitulaire, avec laquelle elle communiquait à l'ouest. Si la salle capitulaire romane n'a pas pu être clairement repérée, les fouilles ont permis d'identifier les aménagements architecturaux, mobiliers et ornementaux de celle qui fut reconstruite au XIII ${ }^{\mathrm{e}} \mathrm{s}$. et qui semble avoir subsisté jusqu'aux remaniements des années 1750 . Une section du chap. 7 présente quelques données nouvelles sur le mode de construction de Cluny III, qui complètent ce qui était connu depuis le livre qu'A. Baud avait consacré à ce sujet en 2003; les murs subsistants de la nef (cinq travées du mur gouttereau sud), dégagés après la destruction d'une écurie du haras, ainsi que la coupole du bras sud du grand transept ont en effet pu faire l'objet de nouveaux relevés archéologiques. S'y ajoutent quelques éléments sur le cloître des $\mathrm{XI}^{\mathrm{e}}$ et $\mathrm{XII}^{\mathrm{e}} \mathrm{s}$. glanés grâce à trois sondages dans les galeries septentrionale et orientale du cloître actuel.
L'ensemble est relativement bref : moins de 150 pages, incluant l'introduction et la conclusion (p. 9-152). Les deux chapitres les plus fournis sont ceux de W. Berry sur les fouilles de K. J. Conant et de C. Sapin et F. Henrion sur Cluny II. Sept annexes complètent l'ouvrage. La première, particulièrement précise et documentée, concerne le mobilier céramique (W. Berry, p. 157-171). Les autres présentent brièvement les carreaux de pavement et les enduits peints de la salle capitulaire, les matériaux de construction de la villa et des églises, les fragments sculptés ainsi que les monnaies mis au jour, puis un tableau récapitulatif des analyses radiocarbones (p. 173-201). Une bibliographie nourrie complète le volume (p. 204-217), dont la moitié des titres se réfère à l'annexe de W. Berry sur la céramique.

Six ans après la fin de la dernière campagne, ce livre était attendu et il livre des résultats remarquables. Les vestiges de la villa ayant précédé le monastère constituent probablement la découverte la plus importante effectuée à Cluny depuis les travaux de K. J. Conant, non seulement parce qu'ils permettent d'éclairer les conditions dans lesquelles le monastère a été créé, mais aussi parce qu'ils apportent quelque lumière au dossier encore obscur de la configuration architecturale et spatiale de l'habitat des élites avant le temps des mottes. La villa de Guillaume d'Aquitaine (appelée bizarrement domus villae par les auteurs, alors qu'aucun texte n'emploie cette expression) apparaît comme un ensemble de murs construits majoritairement en opus spicatum, qui se recoupent à angle droit et qui permettent de restituer le plan d'un complexe architectural dont la surface totale est estimée à $460 \mathrm{~m}^{2}$. La villa aurait été composée d'une dizaine de pièces organisées autour d'une cour rectangulaire $(11,60 \times 8,50 \mathrm{~m})$, sur lesquelles se greffait, dans l'angle-sud-est, une chapelle à nef unique et abside semi-circulaire $(10,40 \times 5,20 \mathrm{~m})$. Des vestiges de planchers en bois, des céramiques et deux monnaies permettent de supposer une édification de la villa au milieu du VIII ${ }^{\mathrm{e}} \mathrm{s}$. et sa destruction progressive au cours de la première moitié $d u \mathrm{x}^{\mathrm{e}} \mathrm{s}$. à mesure de la réorganisation du site par les moines. Sur le plan architectural, elle trouve des parallèles avec plusieurs demeures aristocratiques datées entre le $\mathrm{VII}^{\mathrm{e}}$ et le $\mathrm{X}^{\mathrm{e}} \mathrm{s}$. : C. Sapin retient celles du Thier d'Olne ou de Pouthumé. Elle invite aussi à poursuivre la comparaison, en « aval », avec des demeures d'élites rurales comme celle découverte par François Gentili à Serris-les-Ruelles et, en « amont » avec les palais carolingiens encore mal connus en dehors des résidences impériales les plus prestigieuses. Sur le plan spatial, deux caractéristiques des curtes ou villae 
carolingiennes ont été mises en évidence par les fouilles archéologiques de ces deux ou trois dernières décennies : l'organisation des bâtiments autour d'une cour et la situation fréquente des édifices à proximité de cours d'eau, voire dans des zones marécageuses. Il faudra désormais ajouter Cluny à la série. La question des sites humides mériterait sans doute que l'on s'y attarde. Les fouilles archéologiques menées sous Cluny III dans les années 1990 avaient montré à quel point le site avait été travaillé par les alluvions de la rivière et comment de considérables terrassements avaient été nécessaires pour implanter la maior ecclesia. Les fouilles de la chapelle SainteMarie ont mis au jour un mur interprété comme un éventuel terrassement pour contrer la forte pente en direction de la rivière et, peut-être, protéger les constructions des inondations. L'annexe sur les céramiques montre que les couches les plus anciennes contiennent des tessons datant de la période galloromaine ou des $\mathrm{VI}^{\mathrm{e}}-\mathrm{VII}{ }^{\mathrm{e}} \mathrm{S}$. montrant des traces d'érosion probablement dues à des transports par l'eau. Il serait sans doute intéressant de lier ces différents fils.

En outre, les fouilles menées à Cluny ces dernières années montrent à quel point des recherches doivent encore être entreprises pour comprendre l'espace social dans lequel les moines se sont installés au début $\mathrm{du} \mathrm{x}^{\mathrm{e}} \mathrm{s}$. L'un des trous noirs de la recherche est l'évolution de l'occupation du sol dans la région depuis l'Antiquité. Les présentes fouilles ont mis au jour des céramiques de la période gallo-romaine (point culminant dans la $2^{\mathrm{e}}$ moitié du $\mathrm{II}^{\mathrm{e}}$ et au $\mathrm{III}^{\mathrm{e}} \mathrm{s}$.) et du haut Moyen Âge $\left(\mathrm{VI}^{\mathrm{e}}-\mathrm{X}^{\mathrm{e}}\right)$ qui représentent $39 \%$ de l'ensemble des tessons datables sortis du sol. Ce n'est pas rien. Plus précisément, les analyses de W. Berry montrent une chronologie discontinue : des tessons de production locale pour la période comprise entre le milieu du vie et la seconde moitié du vII ${ }^{\mathrm{e}}$, provenant majoritairement d'un remblai situé sous la villa et attestant d'une occupation sur un site voisin non identifié; une absence apparente de céramique entre la $2^{\mathrm{e}}$ moitié du $\mathrm{VII}^{\mathrm{e}}$ et le milieu du VIII ${ }^{\mathrm{e}} \mathrm{s}$, , puis de nouveau une présence de céramique de provenance principalement locale dans les couches de la villa, dont les tessons sont moins roulés et moins érodés que ceux de la période précédente, et qui plaident pour une continuité de l'occupation depuis le milieu du VIII ${ }^{\mathrm{e}} \mathrm{s}$. Les moines s'installent donc au $\mathrm{x}^{\mathrm{e}} \mathrm{s}$. dans un lieu qui n'est pas neutre et dans un réseau déjà fortement structuré. Quel est-il et comment a-t-il évolué sur la longue durée? Des villae gallo-romaines, des nécropoles du haut Moyen Âge, des églises rurales construites au $\mathrm{VI}^{\mathrm{e}}-\mathrm{VII}^{\mathrm{e}} \mathrm{S}$., ou au $\mathrm{X}^{\mathrm{e}}$, sont connues dans les environs de Cluny. La villa de Cluny, dont on aperçoit désormais quelques éléments de la configuration architecturale, n'a de sens qu'au sein des structures de domination de la région; de même que le monastère, qui prend sa place, n'a de sens qu'au sein du réseau aristocratique dans lequel se situent les premiers abbés (Isabelle Rosé, Construire une société seigneuriale : itinéraire et ecclésiologie de l'abbé Odon de Cluny, fin du IX $X^{e}-$ milieu du $x^{e}$ siècle, Turnhout, Brepols [Collection du Centre d'études médiévales de Nice, 8], 2008) et dans le profond essor économique - et donc architectural - qui a caractérisé le Mâconnais pendant tout le $\mathrm{x}^{\mathrm{e}} \mathrm{s}$. (Alain Guerreau, Saint-Philibert de Tournus : la société, les moines, l'abbatiale, Paris, Association pour l'inscription de l'abbaye Saint-Philibert de Tournus, 2019). Bien sûr, il ne revenait pas au présent ouvrage de mener une telle enquête. Disons que certains de ses résultats appellent à poursuivre la recherche dans ce sens.

Les premiers temps de l'implantation monastique à Cluny apparaissent sous un jour plus concret grâce à ce livre, bien que quelques éléments chronologiques appellent des précisions. Les moines s'installent en 909 ou 910 dans la villa du duc d'Aquitaine. Aucune transformation majeure ne semble être intervenue dans les premiers temps; la chapelle de la villa semble avoir été utilisée telle quelle par les moines. Un mur repéré uniquement en fondation, à l'est de l'abside de la chapelle, a incité les archéologues à supposer un remaniement du sanctuaire pour lui donner la forme d'un chevet plat un peu plus grand que l'abside antérieure, mais il n'a pas été permis de dire si ce chantier a abouti avant que la chapelle de la villa soit détruite. Le terminus ante quem de sa destruction est le règne du roi Louis IV (936-954) dont une monnaie a été retrouvée dans les niveaux correspondant à son abandon et à la construction, à la place, d'un nouvel édifice (la première chapelle Sainte-Marie). Ces observations laissent un peu le lecteur sur sa faim. L'hypothèse d'un chevent plat remplaçant une abside semi-circulaire est d'ailleurs un peu étrange au regard de ce que l'on sait de l'évolution des églises rurales du haut Moyen Âge, qui montre généralement l'inverse.

Ces découvertes laissent également en suspens la question de ce que K. J. Conant appelait « Cluny I », soit la première église abbatiale construite pour les besoins spécifiques du monastère. Sur la base de quelques chartes de Cluny datées entre 912 et 926 qui portent la mention « sacrosanctae aecclesiae sanctorum apostolorum Petri et Pauli quae construitur/que est constructa », K. J. Conant avait supposé une activité constructrice dans le monastère dès les tout premiers temps, ce qui n'aurait en 
effet rien d'étonnant. Il avait également déduit d'un passage de la Vie d'Odon par Jean de Salerne qu'une première église aurait été consacrée à Cluny en 927. C'est celle-ci qu'il associait à " Cluny I » et qu'il localisait, sur des bases ténues, à l'ouest de la villa, tout près du secteur qui accueillit ultérieurement Cluny III. Dans le livre qu'elle a consacré à l'abbatiat d'Odon en 2008, Isabelle Rosé a montré que les textes sollicités par K. J. Conant ne pouvaient pas être lus pour attester la construction ni la dédicace d'une église dans le monastère, et que la date de 927 n'avait aucune pertinence historique (I. Rosé [op. cit. supra], p. 226-231); malheureusement, son livre n'a pas été utilisé par A. Baud et C. Sapin. Par ailleurs, les fouilles des années 1990 ont invalidé l'existence archéologique de « Cluny I » là où l'avait situé K. J. Conant. Autrement dit, il n'en reste rien, du moins dans l'espace connu par les fouilles. Mais les auteurs du présent ouvrage ne le disent pas aussi clairement. Cluny I existe toujours, " consacrée par l'évêque de Mâcon en 927 » et C. Sapin cherche (p. 65) à l'identifier éventuellement à la chapelle de la villa ou au premier état de «Cluny II ». En l'état actuel des connaissances, il conviendrait plutôt d'évoquer l'absence de toute construction d'église par les moines avant celle que l'on appelle Cluny II, de ne plus parler d'une dédicace en 927 et, ce qui serait sans doute le plus sage, d'abandonner définitivement la nomenclature de K. J. Conant (Cluny I, Cluny II et Cluny III) au profit d'une autre, qui corresponde à l'état des connaissances archéologiques. Les auteurs du présent ouvrage le disent timidement (p. 82), mais ils s'arrêtent en chemin, et leur phasage chronologique précis (Cluny IIa, Cluny IIb et Cluny IIc) reste tributaire d'une vision dépassée.

La chronologie de Cluny II mérite aussi quelques précisions. K. J. Conant datait sa construction entre 948 et 981, sur la base de quelques textes. La date de 948 est une déduction à partir d'une charte de donation d'un curtil près de Matour, écrite dans un latin approximatif, qui commence par la mention suivante : «In nomine Domini. Incipit ecclesia Sancto Petro apostolo, cujus est Cluniaco monasterio » (Charte de Cluny $\mathrm{n}^{\circ} 714$, original perdu, connu par sa copie $\mathrm{du} \mathrm{XVIII}^{\mathrm{e}} \mathrm{s}$.). K. J. Conant lisait cet incipit ecclesia comme «l'écho d'une cérémonie qui marque le début juridique, financier ou matériel de Cluny II » (p. 54). L'introduction d'une charte de Cluny par la formule incipit est rare. Il n'en existe que trois autres occurrences avant l'an Mil (2 chartes originales, une copie dans le cartulaire A), et elles s'apparentent à un titre : « incipit donacione 》 (charte 1722), « incipit elemozine » (charte 1799), « incipit noticia » (charte 2076).
Je ne vois dans la charte 714 rien d'autre qu'une annonce maladroite d'une donation à l'église SaintPierre de Cluny. Aussi, serait-il plus prudent de ne pas retenir la date de 948 dans la chronologie de l'abbatiale. Les auteurs du présent ouvrage la mentionnent prudemment (p. 87), pour finalement proposer un premier état de Cluny II au « milieu du $\mathrm{X}^{\mathrm{e}} \mathrm{s}$. » (p. 106), ce qui revient au même. Pourtant, leurs analyses archéologiques plaident pour un aménagement progressif de la villa, par étapes, qui n'exclurait pas une mise en chantier plus précoce. Les hypothèses de C. Sapin sont ici très prudentes, dans la mesure où les vestiges attribués à ce Cluny IIa sont extrêmement ténus : deux fragments de mur, nettement plus larges que ceux de la villa qu'ils remplacent $(1,10 \mathrm{~m}$ au lieu de 0,60 à $0,80 \mathrm{~m}$ ) et construits différemment (l'opus spicatum, qui caractérise l'essentiel des murs de la villa, n'est plus utilisé).

Les parties occidentales et l'origine de la chapelle Sainte-Marie (objet du chap. 6), sont désormais bien connues. Les fouilles archéologiques situent assez clairement la construction de la première chapelle dans le cadre des réaménagements de la villa ayant suivi l'installation des moines, mais la reconstruction complète de Sainte-Marie II, selon un plan plus de deux fois plus grand et un chevet tripartite, reste mal datée. Les auteurs la situent « dans la seconde moitié du XI $\mathrm{I}^{\mathrm{e}} \mathrm{s}$. » (p. 118), « dans le troisième tiers du XI ${ }^{\mathrm{e}} \mathrm{s}$. » (p. 127), « vers 1083-1085» (p. 129), puis en 1085 (p. 151). Tout augmente! Cette date est suggérée par la mention de la dédicace de l'ecclesia infirmorum de Cluny par Eudes (ou Odon), cardinal évêque d'Ostie, dans le récit de la translation des reliques de saint Maximin à Nantua (le texte daterait du XII ${ }^{\mathrm{e}}$ s., il n'est connu que par son édition dans la $P L$, d'après un manuscrit de Nantua aujourd'hui perdu). Sur la base de différents recoupements textuels, N. Stratford avait associé l'ecclesia infirmorum à la chapelle SainteMarie, et daté sa dédicace de septembre 1085, qui correspond au passage d'Eudes à Cluny dans le cadre d'une légation pontificale. Rappelons qu'Eudes est l'ancien grand-prieur de Cluny, qu'il a quitté pour rejoindre la Curie de Grégoire VII, et qui devint pape sous le nom d'Urbain II en 1088. Le récit hagiographique de Nantua ne dit donc rien d'autre que les moines de Cluny ont profité du passage d'un légat pontifical membre de la famille pour dédicacer une église. Il me semble risqué d'en déduire des données précises sur l'état d'achèvement de celle-ci. On sait, depuis les travaux de René Crozet, qu'il faut se garder d'associer trop vite une date de dédicace à une date de construction, surtout lorsque la dédicace n'est pas célébrée par l'évêque ordinaire. Les papes et ses 
légats des temps grégoriens ont en effet consacré de nombreux lieux au cours de leurs voyages, que ce soit des églises complètes, des édifices à peine ébauchés, voire un terrain appeler à accueillir une église; et on connaît l'exemple de Montierneuf de Poitiers où l'on brisa un autel pour en refaire un neuf afin de le faire consacrer par Urbain II. Il conviendrait donc d'être prudents quant à la datation de Sainte-Marie II en dehors de critères archéologiques sûrs. Par ailleurs, si la chapelle Sainte-Marie est bien l'ecclesia infirmorum, comme plusieurs indices textuels semblent le signifier, comment peut-on envisager les circulations entre l'infirmerie et la chapelle? Celle-ci - les fouilles l'ont confirmé - communiquait avec la salle capitulaire, à l'ouest, alors que l'infirmerie se situait à l'est. Il faut donc probablement envisager une autre porte dans la chapelle, mais l'emprise des fouilles ne permettait pas de le vérifier. D'autre part, A. Baud a mené une analyse détaillée des usages liturgiques de la chapelle, notamment dans le cadre des processions ou des offices funéraires (p. 124-127). Ces analyses sont fondées principalement sur le Liber tramitis, coutumier clunisien composé v. 1030. Les rites semblent s'accorder assez bien avec la chapelle telle qu'elle est connue dans son deuxième état, mais celui-ci est daté par A. Baud du $3^{\mathrm{e}}$ tiers du XI ${ }^{\mathrm{e}} \mathrm{s}$. N'y a-t-il pas, dès lors, un problème chronologique?

Terminons ce compte rendu par quelques regrets dus à des maladresses d'ordre formel et structurel, qui laissent un goût d'inachevé. Les chapitres ne sont pas toujours bien articulés les uns aux autres, comme s'il avait manqué un travail de polissage entre les différentes collaborations. Les sources écrites sont évoquées dans les chap. 1, 3 et 5 , et il faut naviguer entre les sections pour saisir l'ensemble des problèmes. Dans les plans récapitulatifs des différents états du site, un code de couleur a été utilisé pour distinguer visuellement les périodes. C'est très pratique, mais cela l'aurait été davantage si ce code avait été expliqué et si son usage avait été constant... On comprend au fil de la lecture que le rouge renvoie à l'état de la villa, que le bleu caractérise le temps de Cluny II ( $\mathrm{x}^{\mathrm{e}}$ et première moitié $\left.\mathrm{XI}^{\mathrm{e}}\right)$, que le violet signale le XII et le brun le XIII ${ }^{\mathrm{e}}$ s., mais dans le chap. 7, le bleu est utilisé pour les élévations de Cluny III, alors que cette couleur est ailleurs réservée à Cluny II. Et le vert reste indécis. Il est utilisé pour caractériser deux murs seulement : la reprise du chevet de la chapelle de la villa, que l'analyse situe dans la première moitié du $\mathrm{X}^{\mathrm{e}} \mathrm{s}$., et un mur situé au chevet de la première chapelle Sainte-Marie, qu'A. Baud interprète comme un possible mur de terrassement, contemporain de la chapelle (donc milieu $\mathrm{X}^{\mathrm{e}}$ ). S'il est contemporain de la chapelle, il devrait figurer en bleu, comme celle-ci. C'est le cas dans la fig. 7 de la p. 114, mais dans la fig. 8, voisine, il est en vert, comme sur les figures suivantes et dans celles du chap. 3 . La confusion s'installe.

Si les plans des différents états de la villa, de Cluny II et de Sainte-Marie sont clairs et précis, ils auraient mérité d'être présentés en plein page. En outre, il faut naviguer dans les chapitres pour comprendre l'évolution progressive du site. Un récapitulatif de tous les états au début ou à la fin de l'ouvrage aurait été nécessaire, ainsi que l'ajout d'un ou de plusieurs plans permettant de comprendre comment les édifices se succèdent au même emplacement. Il faut par exemple se faire son propre plan mental pour comprendre comment et où le chevet de Cluny II, dans son état développé, s'implante par rapport à l'ancienne villa.

La mise en page est parfois un peu perturbante : certaines figures sont présentées dans le désordre, le texte est interrompu entre les p. 69 et 75 par un encadré qui aurait pu facilement être placé à la fin $\mathrm{du}$ chapitre. Les illustrations sont parfois petites et difficiles à lire, en particulier les relevés en plan ou en élévation (celles des élévations de la chapelle Sainte-Marie, p. 116-117 sont en format timbreposte). Les coupes stratigraphiques qui figurent dans les différents chapitres ne bénéficient pas toutes de la même précision. Celles des sections sur Cluny II sont accompagnées de légendes qui permettent de comprendre à quoi renvoie la couche 38 ou le mur 74, mais pas celles du chapitre sur Sainte-Marie. Il faut alors faire preuve de patience pour retrouver sur les plans récapitulatifs les numéros des murs et des couches associées. La section sur la chapelle Sainte-Marie concentre d'ailleurs les coquilles et imperfections : les figures signalées p. 111 (fig. 1 et 4) présentent autre chose que ce qui est dit dans le texte; on lit Sainte-Marie I au lieu de Sainte-Marie II dans la légende de la fig. 11 (p. 115); une note est répétée deux fois (n. 5 et n. 22); la maior ecclesia est située à la fin du $x^{\mathrm{e}} \mathrm{s}$. (p. 124).

En définitive, en dehors des deux chapitres dus à Walter Berry qui sont très clairs et extrêmement documentés, ce livre nécessite une certaine patience pour suivre la pensée des auteurs. C'est dommage pour des résultats aussi importants, qui « dépassent la simple problématique clunisienne », comme l'écrit C. Sapin à la fin du chapitre consacré à la villa (p. 61). Les fouilles se poursuivent aujourd'hui à Cluny sous la direction d'A. Baud, dans un secteur situé plus à l'est, à l'emplacement présumé de l'infirmerie médiévale. Il faut souhaiter que les secteurs étudiés entre 2006 et 2013 n'en restent pas là et que l'on puisse poursuivre la réflexion sur les antécédents, les origines et le premier Cluny.

Didier MÉHU

Université Laval (Québec) 